\title{
Meshing scheme in the computation of spontaneous fault rupture process ${ }^{*}$
}

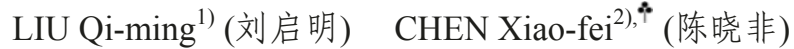 \\ 1) School of Earth and Space Science, Peking University, Beijing 100871, China \\ 2) School of Earth and Space Science, University of Science and Technology of China, Hefei 230026, China
}

\begin{abstract}
The choice of spatial grid size has been being a crucial issue in all kinds of numerical algorithms. By using BIEM (Boundary Integral Equation Method) to calculate the rupture process of a planar fault embedded in an isotropic and homogeneous full space with simple discretization scheme, this paper focuses on what grid size should be applied to control the error as well as maintaining the computing efficiency for different parameter combinations of $\left(D_{\mathrm{c}}, T_{\mathrm{e}}\right)$, where $D_{\mathrm{c}}$ is the critical slip-weakening distance and $T_{\mathrm{e}}$ is the initial stress on the fault plane. We have preliminarily found the way of properly choosing the spatial grid size, which is of great significance in the computation of seismic source rupture process with BIEM.
\end{abstract}

Key words: seismic source dynamics; finite fault source rupture; boundary integral equation; meshing scheme CLC number: P $315.3^{+} 3 \quad$ Document code: A

\section{Introduction}

Numerical simulations of seismic source dynamics have great significance in understanding both the physical mechanisms and the radiation characteristics of the rupture processes. The numerical methods widely used in this field include the Finite Difference Method (FDM) (Madariaga, 1976; Andrews, 1976), the Finite Element Method (FEM) (Oglesby et al, 2000; Aagaard (et al, 2001) and the Boundary Integral Equation Method (BIEM) (Fukuyama and Madariaga, 1995; Aochi et al, 2000). Among these methods, BIEM gets much attention for its efficiency and accuracy (Aochi et al, 2000; Zhang and Chen, 2006), and plays an important role in the study of seismic source dynamics.

BIEM is a semi-numerical and semi-analytical method. In principle, the integral equation which controls the changes of stress and slip on the fault plane is firstly obtained based on representation theorem, then discretized into a set of linear equations and finally solved by incorporating with a friction law. Generally speaking, the smaller the grid size is, the more accurate the simulation will be. However, the cost of simulation will increase significantly when the grid size decreases. Thus, tradeoff between efficiency and accuracy needs to be made, which means that a maximum grid size in accordance with a given error level should be found. Assuming that all the

\footnotetext{
* Received 2008-05-30; accepted in revised form 2008-06-12.

Foundation item: National Natural Science Foundation of China (40504004 and 40521002).

^Author for correspondence: xfchen1@ustc.edu.cn
} 
stresses are measured relatively to the residual stress and a slip-weakening law is applied, the allowed maximum grid size is dependent on basic parameters such as critical slip-weakening distance $D_{\mathrm{c}}$, shear strength $T_{\mathrm{u}}$ and initial stress $T_{\mathrm{e}}$. Although the determination of the grid size is important, few studies on this problem have been carried out so far, which limits the applications of BIEM. In this article, we performed twelve selected simulations with various $D_{\mathrm{c}}$ and $T_{\mathrm{e}}$ for a given error level of $2 \%$, and revealed the dependence of maximum grid size on $D_{\mathrm{c}}$ and $T_{\mathrm{e}}$ by comparisons between different simulations.

\section{Model configuration}

For simplicity, we discuss the dynamic rupture processes on a finite planar fault embedded in an unbounded, homogeneous and isotropic elastic medium. As shown in Figure 1, a $6 \mathrm{~km} \times 6 \mathrm{~km}$ asperity at the center of a $24 \mathrm{~km} \times 24 \mathrm{~km}$ square fault is the rupture nucleation zone. A simple box discretization scheme (Aochi et al, 2000; Zhang and Chen, 2006) is applied, in which the slip rate between two adjacent time steps in any spatial grid remains a constant. $A, B$ and $C$ are three "observation points" used for evaluating the accuracy of the simulations with different parameters and grid sizes.

By taking partial derivatives of both sides of the displacement representation theorem in frequency domain (Aki and Richards, 2002), we can get the stress representation theorem as follows

$$
\widetilde{\tau}_{k l}(\boldsymbol{x}, \omega)=c_{k l n m} \iint_{\Sigma} \Delta \widetilde{u}_{i}(\xi, \omega) c_{i j p q} v_{j} \frac{\partial}{\partial \xi_{m}}\left[\widetilde{G}_{n p, q}(x, \xi, \omega)\right] \mathrm{d} S(\xi)
$$

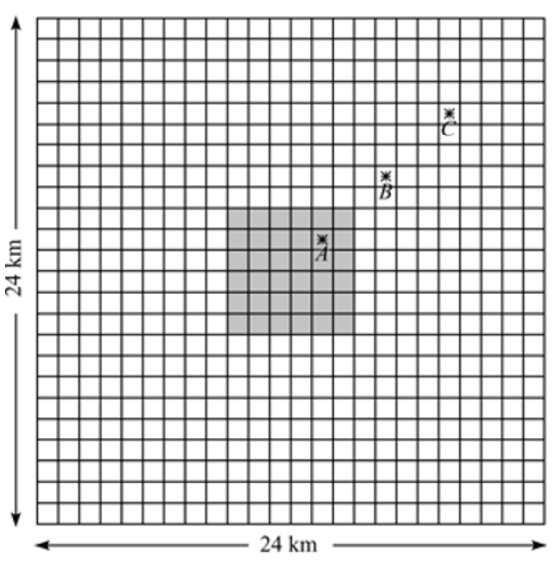

Figure 1 Geometry model for this study and the three observing points

In equation (1), $\xi$ and $\boldsymbol{x}$ are coordinates of source and field points, respectively. $\Sigma$ represents the two sides of the fault plane, $\omega$ is the circular frequency, $\Delta \widetilde{u}_{i}$ is the dislocation on the fault, $\widetilde{G}_{n p, q}$ is the spatial derivatives of the Green's function. $c_{i j p d}$ and $v_{j}$ are the elastic constants of the medium and the unit vector normal to the fault plane, respectively. After discretization of the integral equation, the problem of spontaneous rupture is transformed into a set of linear equations. Incorporated with a friction law, these equations can be solved iteratively step by step. The most widely used friction law is the slip-weakening law (Ida, 1972) shown in Figure 2. An appropriate Courant-Friedrichs-Lewy (CFL) should be applied in the simulation to keep the computation stable (Madariaga et al, 1998).

In order to investigate how different combinations of $D_{\mathrm{c}}$ and $T_{\mathrm{e}}$ affect the choice of the maximum grid size, three typical values of $D_{\mathrm{c}}$ are selected in the simulation: $0.070 \mathrm{~m}$ (small), $0.348 \mathrm{~m}$ (middle) and $0.695 \mathrm{~m}$ (large). For a specific $D_{\mathrm{c}}$ there is a critical value $T_{\mathrm{ec}}\left(D_{\mathrm{c}}\right)$, only when $T_{\mathrm{e}}>T_{\mathrm{ec}}\left(D_{\mathrm{c}}\right)$ the rupture can take place in the entire computational domain. Therefore, we will determine the $T_{\mathrm{ec}}\left(D_{\mathrm{c}}\right)$ through numerical tests first, then with this value as the starting point, we will select three other values of $T_{\mathrm{e}}$. Other parameters remain fixed in all the simulations, such 
as $C F L=0.4$, the shear strength $T_{\mathrm{u}}=10 \mathrm{MPa}$, the initial stress within the asperity $T_{\mathrm{i}}$ $=10.01 \mathrm{MPa}$, the density $\rho=3.30 \mathrm{~g} / \mathrm{cm}^{3}$, Possion's ratio $v=0.25$, Young's modulus $E=3.45 \times 10^{+10} \mathrm{~Pa}$. We ran 12 simulations with three different $D_{\mathrm{c}}$ and four distinct $T_{\mathrm{e}}$. For each set of instances, we apply a gradually refined meshing scheme. The computation domain shown in Figure 1 is divided into grids of $24 \times 24(\Delta s=1 \mathrm{~km})$, $40 \times 40(\Delta s=0.6 \mathrm{~km}), 56 \times 56(\Delta s=0.43 \mathrm{~km})$, $72 \times 72(\Delta s=0.33 \mathrm{~km}), 88 \times 88(\Delta s=0.27 \mathrm{~km})$, $104 \times 104(\Delta s=0.23 \mathrm{~km}), \cdots,[4(6+4 n)] \times$

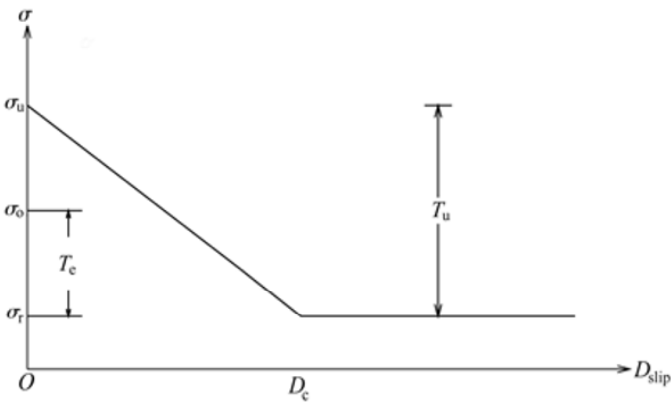

Figure 2 Sketch for the slip-weakening law $x$ and $y$ axis denote slip and stress, respectively. $\sigma_{\mathrm{u}}$ is the peak strength, $\sigma_{0}$ is the absolute initial stress, $\sigma_{\mathrm{r}}$ is the residual stress, and $D_{\mathrm{c}}$ is the critical slip-weakening distance $[4(6+4 n)] .(\Delta s=3 /[3+2 n]), \cdots$. We define the scheme with grid of $[4(6+4 n)] \times[4(6+4 n)]$ as the "scheme $n$ " for short hereafter. These gradually refined schemes can guarantee that the "observation points" $A, B$ and $C$ always stay at the center of the corresponding grid. In other words, the absolute locations of the three selected "observation points" remain exactly the same for all meshing schemes, thus the results obtained in different meshing schemes could be fairly compared.

To quantitatively evaluate the accuracy of the simulation results, we define the error function for the simulation result with scheme $n$ as follows

$$
E_{\text {error }}(n)=\frac{t_{n}-t_{n-1}}{t_{n-1}} \quad(n=1,2, \cdots, 8)
$$

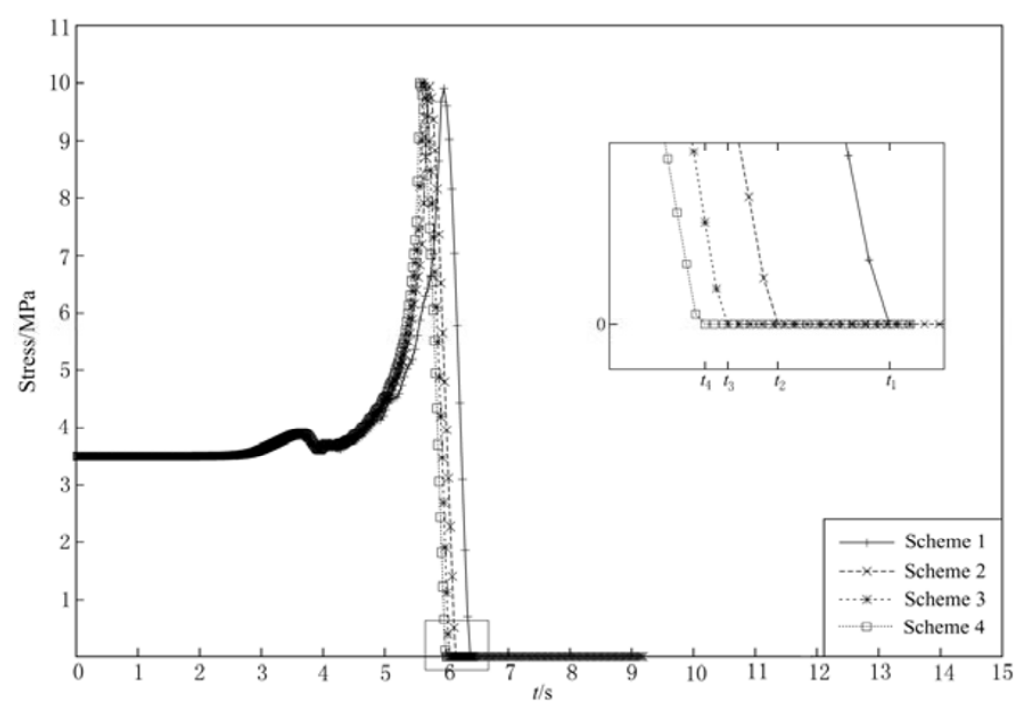

Figure 3 Stress as a function of time at observation point $C$ (see Figure 1) in four different meshing schemes with $\left(D_{\mathrm{c}}=0.348 \mathrm{~m}, \bar{T}_{\mathrm{e}}=0.36\right)$

The rectangular zone below is zoomed into the subfigure in the right corner, in which $t_{n}(n=1,2,3,4)$ are the characteristic time values in "scheme- $n$ " defined in the text, respectively 
Here $n$ represents "scheme $n$ " and $t_{n}$ is a characteristic value of a real-time variable measured at the observation points. For convenience we here use point $C$ and designate the time when its stress drops to 0 as $t_{n}$. Such definition has clear physical meaning and is easy to implement. Moreover, good correlation between this characteristic time and relative errors of several other physical quantities is also found in our simulations. Hence, it is reasonable to use this definition in error analysis. Figure 3 demonstrates the error analysis.

\section{Numerical results with di fferent combinations of $D_{\mathrm{c}}$ and $T_{\mathrm{e}}$}

First, we determine the $T_{\mathrm{ec}}$ corresponding to three different $D_{\mathrm{c}}$ through numerical tests. For the dimensionless initial shear stress $\bar{T}_{\mathrm{e}}\left(=T_{\mathrm{e}} / T_{\mathrm{u}}\right)$, the accuracy of our tests is chosen as 0.02 . As shown in Table 1, the dimensionless characteristic value $\bar{T}_{\mathrm{ec}}$ is the average of two adjacent values of $\bar{T}_{\mathrm{e}}$ which generate two distinct types of rupture processes, i.e., the "non-growth rupture" and "rupture". Figures $4 \mathrm{a}$ and $4 \mathrm{~b}$ show the snapshots of the rupture processes when $\bar{T}_{\mathrm{e}}=0.28$ and $\bar{T}_{\mathrm{e}}=0.30$ respectively (with $D_{\mathrm{c}}=0.348 \mathrm{~m}$ ). It is obvious that when $\bar{T}_{\mathrm{e}}=0.28$ the rupture process stops shortly after its nucleation, which is called "non-growth rupture". For $\bar{T}_{\mathrm{e}}=0.30$ the rupture keeps propagating outward and called "rupture". Although the difference between the two values of $\bar{T}_{\mathrm{e}}$ is only 0.02 , the rupture processes are totally different. Figures $5 \mathrm{a}$ and $5 \mathrm{~b}$ show the evolutions of slip and stress at the observation points $A, B$ and $C$ in each case. Comparing the slip and stress curves of point $C$ in Figures $5 \mathrm{a}$ and $5 \mathrm{~b}$, we can see that with $\overline{T_{\mathrm{e}}}=0.28$, rupture never occurs.

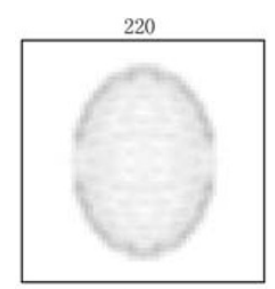

190

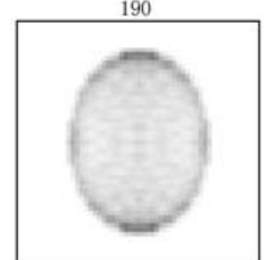

160

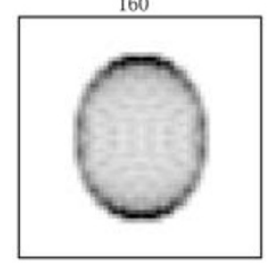

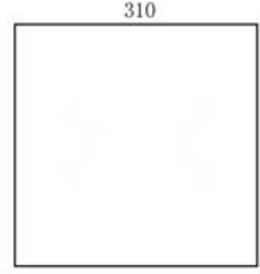

280

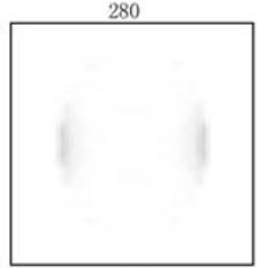

250

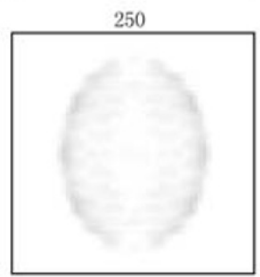

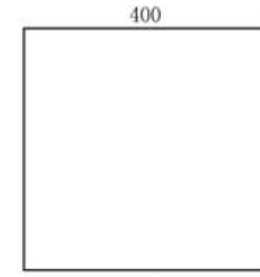

370
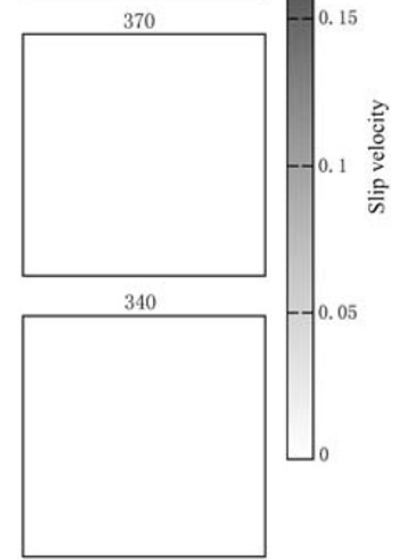

Figure $4 \mathrm{a}$ Snapshot of slip rate (non-dimensional) in the rupture propagation with $D_{\mathrm{c}}=0.348$ $\mathrm{m}$ and $\bar{T}_{\mathrm{e}}=0.28$

Numbers on subfigures indicate time steps 


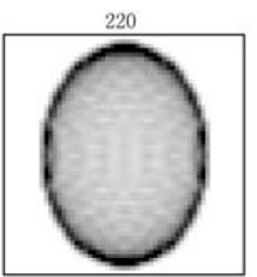

190

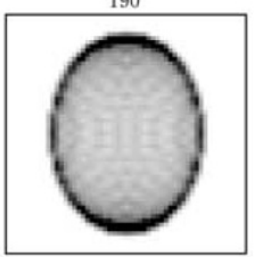

160
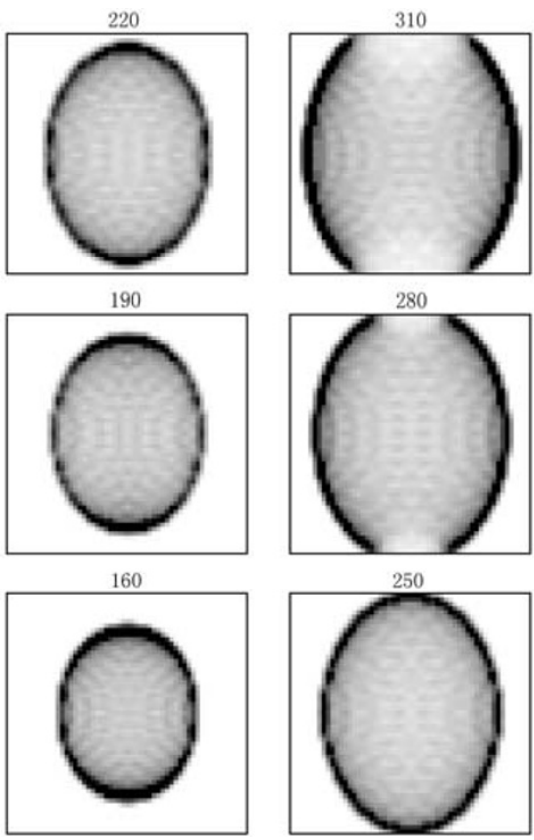

280
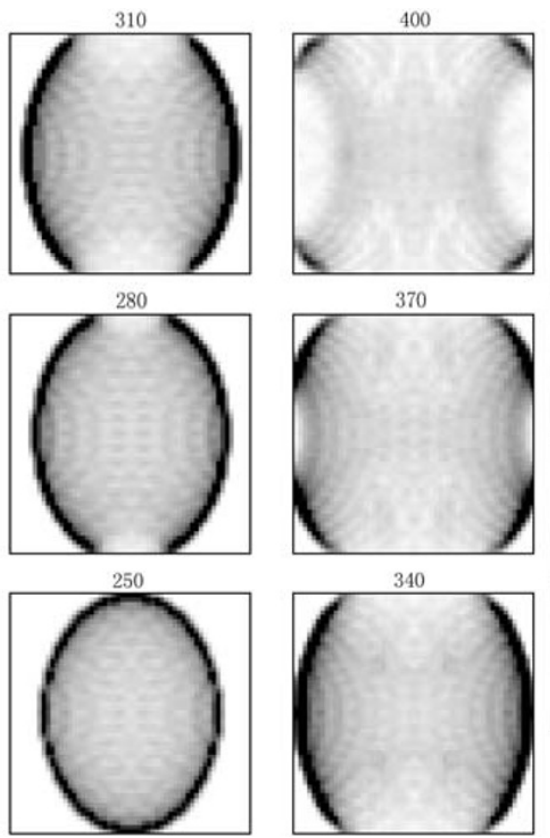

370
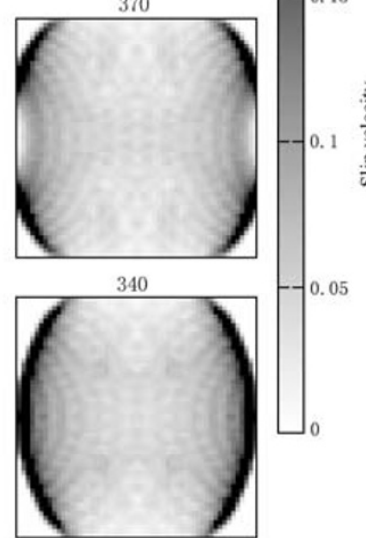

Figure $4 \mathrm{~b}$ Snapshot of slip rate (non-dimensional) in the rupture propagation process with $D_{\mathrm{c}}=0.348 \mathrm{~m}$ and $\bar{T}_{\mathrm{e}}=0.30$

Numbers on subfigures indicate time steps
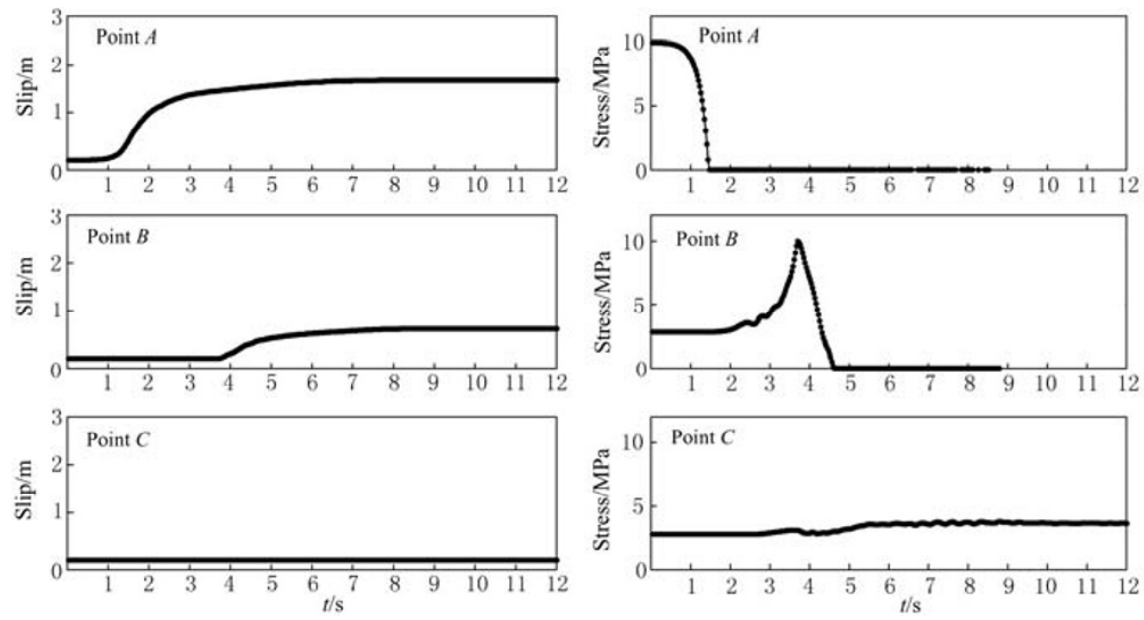

Figure 5a Slip and stress as a function of time at points $A, B$ and $C$ with $D_{\mathrm{c}}=0.348 \mathrm{~m}$ and $\bar{T}_{\mathrm{e}}=0.28$

Next, choosing $2 \%$ as the allowed error, we run a number of simulations for each combination of $D_{\mathrm{c}}$ and $T_{\mathrm{e}}$ by gradually refining the computing grids with the special meshing scheme defined above, and then determine the grid size of the first "scheme $n$ " whose error is below $2 \%$ as the maximum grid size for the corresponding $D_{\mathrm{c}}$ and $T_{\mathrm{e}}$. Figure 6 shows the maximum grid sizes for the twelve combinations of $D_{\mathrm{c}}$ and $T_{\mathrm{e}}$ according to our simulations.

Because the values of $\bar{T}_{\mathrm{ec}}$ for different $D_{\mathrm{c}}$ are different as shown in Table 1, the selected four values of $T_{\mathrm{e}}$ for each $D_{\mathrm{c}}$ are chosen in following way 


$$
\bar{T}_{\mathrm{e} 1}=\bar{T}_{\mathrm{ec}}\left(D_{\mathrm{c}}\right)+0.01, \bar{T}_{\mathrm{e} 2}=\bar{T}_{\mathrm{ec}}\left(D_{\mathrm{c}}\right)+0.06, \bar{T}_{\mathrm{e} 3}=\bar{T}_{\mathrm{ec}}\left(D_{\mathrm{c}}\right)+0.11, \bar{T}_{\mathrm{e} 4}=\bar{T}_{\mathrm{ec}}\left(D_{\mathrm{c}}\right)+0.21
$$

that is, they start from $\bar{T}_{\mathrm{ec}}\left(D_{\mathrm{c}}\right)$.
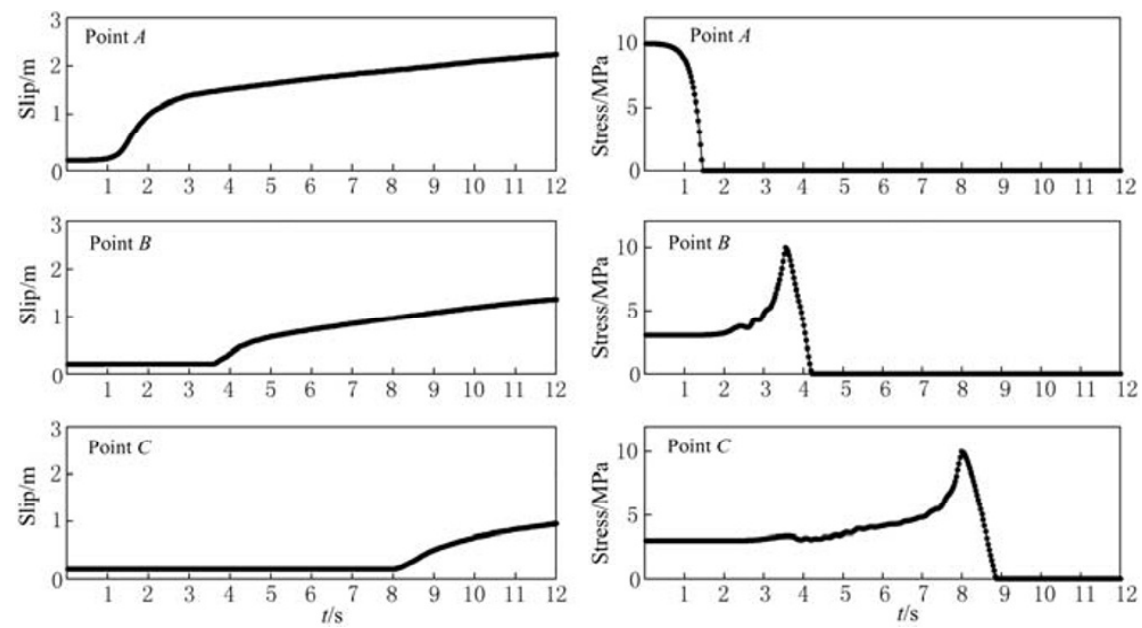

Figure 5b Slip and stress as a function of time at points $A, B$ and $C$ with $D_{\mathrm{c}}=0.348 \mathrm{~m}$ and $\bar{T}_{\mathrm{e}}=0.30$

Table 1 Decision of characteristic value of $\bar{T}_{\mathrm{ec}}\left(D_{\mathrm{c}}\right)$

\begin{tabular}{cccc}
\hline$D_{\mathrm{c}} \mathrm{m}$ & Non-growth rupture $\bar{T}_{\mathrm{e}}$ & Rupture $\bar{T}_{\mathrm{e}}$ & Characteristic value $\bar{T}_{\mathrm{ec}}$ \\
\hline 0.070 & 0.16 & 0.18 & 0.17 \\
0.348 & 0.28 & 0.30 & 0.29 \\
0.695 & 0.47 & 0.49 & 0.48 \\
\hline
\end{tabular}

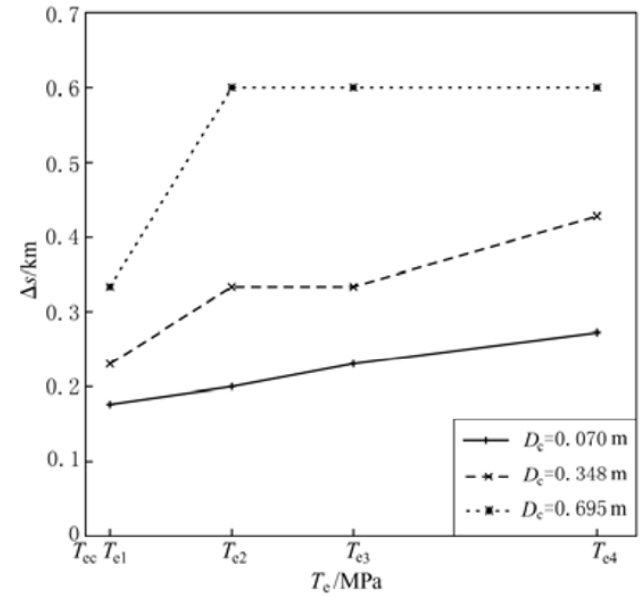

Figure 6 The max $\Delta s$ for different $\left(D_{\mathrm{c}}, T_{\mathrm{e}}\right)$ under a relative error bound $(2 \%)$

$x$ and $y$ axis represent effective stress and $\Delta s$, respectively

\section{Discussion and conclusions}

From Figure 6 we can see that for a fixed $D_{\mathrm{c}}$, the maximum grid size is proportional to $\left(\bar{T}_{\mathrm{e}}-\bar{T}_{\mathrm{ec}}\right)$. Comparisons between different curves in this figure clearly indicate that as $D_{\text {c }}$ decreases all the computational grids should be condensed to ensure sufficient accuracy. These conclusions provide very important and useful guidelines for efficiently and accurately simulating the seismic source rupture processes with BIEM.

Because our meshing scheme is not continuous and only four values of $T_{\mathrm{e}}$ are selected for each $D_{\mathrm{c}}$, the curves in Figure 6 are not smooth enough. Furthermore, since the error of "scheme 0 " could not be computed with equa-

tion (2), our minimum grid division is chosen as $40 \times 40$ (i.e., "scheme 1"), which results in a "saturation" in the simulation meshing scheme shown in Figure 6 in which the curve for $D_{\mathrm{c}}=0.695 \mathrm{~m}$ 
becomes flat.

Acknowledgments We are grateful to Dr. ZHANG Hai-ming for his assistance and the stimulating discussions during the course of this study.

\section{References}

Aagaard B, Heaton T, Hall J. 2001. Dynamic earthquake ruptures in the presence of lithostatic normal stresses: Implications for friction models and heat production [J]. Bull Seism Soc Amer, 91: 1765-1 796.

Aki K and Richards P. 2002. Quantitative Seismology: Theory and Methods (Second Edition) [M]. Sausalito, California: University Science Books: 38-60.

Andrews D. 1976. Rupture propagation with finite stress in antiplane strain [J]. J Geophys Res, 81: 3 575-3 582.

Aochi H, Fukuyama E, Matsu'ura M. 2000. Spontaneous rupture propagation on a non-planar fault in 3-D elastic medium [J]. Pure Appl Geophys, 157: 2 003-2 027.

Fukuyama E and Madariaga R. 1995. Integral equation method for plane crack with arbitrary shape in 3D elastic medium [J]. Bull Seism Soc Amer, 85: 614-628.

Ida Y. 1972. Cohesive force across the tip of a longitudinal-shear crack and Griffith's specific surface energy [J]. J Geophys Res, 77: $3796-3805$.

Madariaga R, Olsen K, Archuleta R. 1998. Modeling dynamic rupture in a 3D earthquake fault model [J]. Bull Seism Soc Amer, 88: 1 182-1 197.

Madariaga R. 1976. Dynamic of an expanding circular fault [J]. Bull Seism Soc Amer, 66: 639-667.

Oglesby D, Archuleta R, Nielson S. 2000. The three-dimensional dynamics of dipping faults [J]. Bull Seism Soc Amer, 90: 616-628.

Zhang H M and Chen X F 2006. Dynamic rupture on a planar fault in three-dimensional half space: I. Theory [J]. Geophys J Int, 164(3): $633-652$. 